INVITED PAPER

\title{
Empirical Mode Decomposition for Advanced Speech Signal Processing
}

\author{
Md. Khademul Islam Molla ${ }^{1,2}$, Somlal Das ${ }^{2}$, Md. Ekramul Hamid ${ }^{2}$ and Keikichi Hirose ${ }^{1}$ \\ ${ }^{1}$ Department of Information and Communication Engineering, The University of Tokyo, Tokyo 113-8656, Japan \\ ${ }^{2}$ Department of Computer Science and Engineering, The University of Rajshahi, Rajshahi 6205, Bangladesh \\ E-mail: ${ }^{1}\{$ molla, hirose\}@gavo.t.u-tokyo.ac.jp
}

\begin{abstract}
Empirical mode decomposition (EMD) is a newly developed tool to analyze nonlinear and non-stationary signals. It is used to decompose any signal into a finite number of time varying subband signals termed as intrinsic mode functions (IMFs). Such data adaptive decomposition is recently used in speech enhancement. This study presents the concept of EMD and its application to advanced speech signal processing paradigms including speech enhancement by soft-thresholding, voiced/unvoiced $(\mathrm{V} / \mathrm{Uv})$ speech discrimination and pitch estimation. The speech processing is frequently performed in the transformed domain and the transformation is usually achieved by traditional signal analysis techniques i.e. Fourier and wavelet transformations. These analysis methods employ priori basis function and it is not suitable for data adaptive analysis for non-stationary signal like speech. Recently, EMD is taken much attention for speech signal processing in data adaptive way. Several EMD based potential soft-thresholding algorithms for speech enhancement are discussed here. The V/Uv discrimination is an important concern in speech processing. It is usually performed by using acoustic features. The training data is used to determine the threshold for classification. The EMD based data adaptive thresholding approach is developed for $\mathrm{V} / \mathrm{Uv}$ discrimination without any training phase. Noticeable improvement is achieved with the application of EMD in pitch estimation of noisy speech signals. The related experimental results are also presented to realize the effectiveness of EMD in advanced speech processing algorithms.
\end{abstract}

Keywords: empirical mode decomposition, pitch estimation, soft-thresholding, speech enhancement, voiced/unvoiced speech classification

\section{Introduction}

Empirical mode decomposition (EMD) is newly developed tool to analyze non-linear and non-stationary signals. Traditional data analysis methods are all based on linear and stationary assumption of the signals. Only in recent years have new methods been introduced to analyze non-stationary and non-linear data. Wavelet analysis and the Wanger-Ville distribution $[1,2]$ are designed for linear but non-stationary data. Additionally, various non-linear time series analysis methods [3-5] are designed for nonlinear but stationary and deterministic systems. In most real systems, either natural or even man-made one, the data are most likely to be both nonlinear and nonstationary. Analyzing the data from such a system is a daunting problem. Even the universally accepted mathematical paradigm of data expansion in terms of a priori established basis would need to be eschewed, for the convolution computation of a priori basis creates more problems than solutions. A necessary condition to represent nonlinear and nonstationary data is to have an adaptive basis. A priori defined function cannot be relied on as a basis, no matter how sophisticated the basis function might be. A few adaptive methods are available for signal analysis, as summarized in [6]. However, the methods given in their book are well designed for nonstationary processes. For nonstationary and nonlinear data, where adaptation is absolutely necessary, no available method can be found. Being adaptive means that the definition of the basis has to be data dependent, an a posteriori defined basis, an approach totally different from the established mathematical paradigm for data analysis. Therefore, the required definition presents a great challenge to the mathematical community. Even though challenging, new methods to examine data from the real world are certainly needed. A recently developed method, empirical mode decomposition (EMD) [7-9] seems to be able to meet the requirement of posterior basis function necessary for adaptive data analysis.

EMD is a method of breaking down a signal without leaving the time domain. It can be compared to other analysis methods like Fourier Transforms and wavelet decomposition. The process is useful for analyzing natural signals, which are most often non-linear and non-stationary. EMD filters out functions which form a complete and nearly orthogonal basis for the original signal. The functions, known as Intrinsic Mode Functions (IMFs), are therefore sufficient to describe the signal, even though they are not necessarily orthogonal [8]. Locally, any two components are orthogonal in term of instantaneous frequency. The fact that the functions into which a signal is decomposed are all in the time-domain and of the same length as the original signal allows for varying frequency 
in time to be preserved. Obtaining IMFs from real world signals is important because natural processes often have multiple causes, and each of these causes may happen at specific time intervals. This type of data is evident in an EMD analysis, but quite hidden in the Fourier domain or in wavelet coefficients.

EMD also has several limitations. The most comprehensive studies on the performance and limitations of EMD with particular applications to irregular waves are performed in [10]. The authors make extensive investigation into the spline interpolation and discuss using additional points, both forward and backward, to determine better envelopes. They also performed a parametric study on the proposed improvement and showed significant improvement in the overall EMD computations. It is limited in distinguishing different components in narrowband signals [11]. If the frequencies of two components are very close, it is difficult to separate them by using EMD. The adaptive method can let the data reveal their underlying process without any undue influence from the basis function. However, no mathematical model exists for such approach.

As mentioned above, EMD is most useful for nonlinear, non-stationary signals, and natural signals. The EMD based approach is widely used in audio signal processing [12, 13], computational neuroscience [14], climate signal analysis [15], image processing [16], seismic signal [17] and biomedical signal processing [18]. This study focuses on the application of EMD in advanced speech signal processing including fundamental frequency estimation [19], voiced/unvoiced speech classification [20] and speech enhancement [21, 22]. The remaining parts of the paper are organized as - the basics of EMD and its limitations are described in Section 2, the EMD based approaches to speech signal processing and enhancement are explained in Section 3 and Section 4 respectively, some experimental results and discussion are illustrated in Section 5 and Section 6 includes the concluding remarks.

\section{EMD Basics}

Empirical mode decomposition (EMD) represents any temporal signal into a finite set of AM-FM oscillating components which are bases of the decomposition. The key benefit of using EMD is that it is an automatic decomposition and fully data adaptive [23]. The principle of the EMD technique is to decompose a signal $s(t)$ into a sum of the band-limited functions $d_{m}(t)$ called intrinsic mode functions (IMFs). Each IMF satisfies two basic conditions: (i) in the whole data set, the number of extrema and the number of zero crossings must be the same or differ at most by one, (ii) at any point, the mean value of the envelope defined by the local maxima and the envelope defined by the local minima is zero. The first condition is similar to the narrow-band requirement for a stationary Gaussian process and the second condition is a local requirement induced from the global one, and is necessary to ensure that the instantaneous frequency will not have redundant fluctuations as induced by asymmetric waveforms.

\subsection{Univariate EMD (uEMD)}

The univariate EMD (uEMD) is used to decompose univariate signals into a finite set of IMFs. There exist many approaches of computing EMD [24]. The following algorithm is employed here to decompose signal $s(t)$ into a set of IMF components.

1. Set $g_{l}(t)=s(t)$

2. Detect the extrema (both maxima and minima) of $g_{I}(t)$

3. Generate the upper and lower envelopes $h(t)$ and $l(t)$ respectively by connecting the maxima and minima separately with cubic spline interpolation

4. Determine the local mean as: $\mu_{l}(t)=[h(t)+l(t)] / 2$

5. IMF should have zero local mean; subtract $\mu_{l}(t)$ from the original signal as: $g_{I}(t)=g_{I}(t)-\mu_{l}(t)$

6. Decide whether $g_{l}(t)$ is an IMF or not by checking the two basic conditions as described above

7. Repeat steps 2 to 6 and end when with an IMF $g_{l}(t)$

Once the first IMF is derived, define $\delta_{I}(t)=g_{I}(t)$, which is the smallest temporal scale in $s(t)$. To find the rest of the IMF components, generate the residue $r_{1}(t)$ of the data by subtracting $\delta_{I}(t)$ from the signal $s(t)$ as: The sifting process will be continued until the final residue is a constant, a monotonic function, or a function with only one maxima and one minima from which no more IMF can be derived.

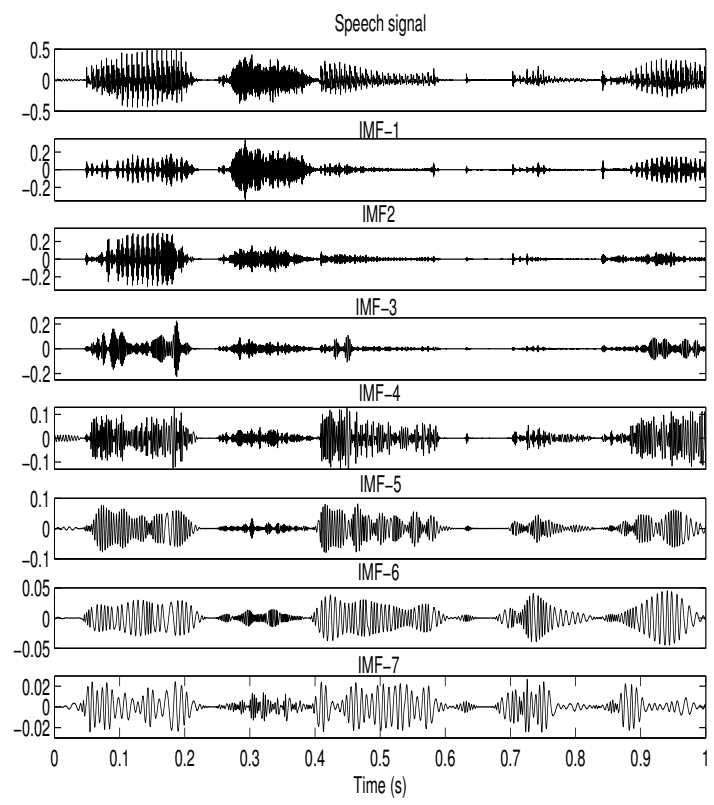

Fig. 1 Univariate EMD (first 7 IMFs out of 13) of speech signal

The subsequent basis functions and the residues are computed as

$$
r_{1}(t)-\delta_{2}(t)=r_{2}(t), \ldots, r_{M-1}(t)-\delta_{M}(t)=r_{M}(t)
$$

where $r_{M}(\mathrm{t})$ is the final residue. At the end of the decomposition the signal $s(t)$ is represented as:

$$
s(t)=\sum_{m} \delta_{m}(t)+r_{R}(t)
$$

where $r_{R}(t)$ is the final residue which can be either the 
mean trend or a constant, and functions $\delta_{m}(t)$ are nearly orthogonal to each other, and all have zero means. The EMD (individual IMF) of speech signal is illustrated in Fig. 1. More specifically, the first component has the smallest time scale which corresponds to the fastest time variation of data. As the decomposition process proceeds, the time scale increases, and hence, the mean frequency of the mode decreases [24]. Since the decomposition is based on the local characteristic time scale of the data to yield adaptive basis, it is applicable to nonlinear and nonstationary data in general and in particular.

\subsection{Instantaneous frequency of IMF}

Instantaneous frequency (IF) represents signal's frequency at any time instance and it is defined as the rate of change of the phase angle at the instant of the "analytic" version of the signal. Every IMF is a real valued signal. The discrete Hilbert transform (dHT) is used to compute the analytic signal for an IMF. Then the analytic version of the $m^{\text {th }}$ IMF $\delta_{m}(t)$ is defined as:

$$
z_{m}(t)=\delta_{m}(t)+j H_{d}\left[\delta_{m}(t)\right]=\gamma_{m}(t) e^{j \theta_{m}(t)}
$$

where $\gamma_{m}(t)$ and $\theta_{m}(t)$ are instantaneous amplitude and phase respectively of the $m^{\text {th }}$ IMF. The discrete Hilbert transform $H_{d}[$.$] is defined as [25]:$

$$
H_{d}\left[\delta_{m}(t)\right]=\frac{1}{\pi} \sum_{\tau=1, \tau \neq t}^{T} \frac{\delta_{m}(\tau)}{t-\tau}
$$

The analytic signal is advantageous in determining the instantaneous quantities such as energy, phase and frequency. The IF of $m^{\text {th }}$ IMF is then given as the derivative of the phase $\theta_{m}(t)$-calculated at $t$ i.e.

$$
f_{m}(t)=\frac{\partial \widetilde{\theta}_{m}(t)}{\partial t}
$$

where $\tilde{\theta}_{m}(t)$ represents the unwrapped version of instantaneous phase $\theta_{m}(t)$. The derivative in Eq. (5), is evaluated at discrete instant of $t$. It should be noted that such derivative introduces the abrupt fluctuations of IF and hence nonlinear smoothing is required. Here, the moving average smoothing filter is used to remove such fluctuations. The filtering scheme improves the effectiveness of computing IF using discrete derivative. The IF of individual IMF shown in Fig. 1 is illustrated in Fig. 2. The concept of IF is physically meaningful only when applied to mono-component signals. In order to apply the concept of IF to arbitrary signals it is necessary to decompose the signal into a series of mono-component contributions. In the recent approaches [8], EMD technique decomposes a time domain signal into a series of monocomponent IMFs. Then the IF derived for each component provides the meaningful physical information.

Although the IMFs may have frequency overlaps but at any time instant, the instantaneous frequencies represented by each IMF are different. This phenomenon can be well understood in Fig. 2 which shows the instantaneous frequencies of the first 6 IMFs of the noisy speech signal shown in Fig. 1. Therefore, EMD is an effective decomposition of non-linear and non-stationary signals in terms of their local frequency characteristics. With such property, each frequency component of the signal is clearly identified and localized in both time and frequency scales yielding the signal spectra at each sampling point.

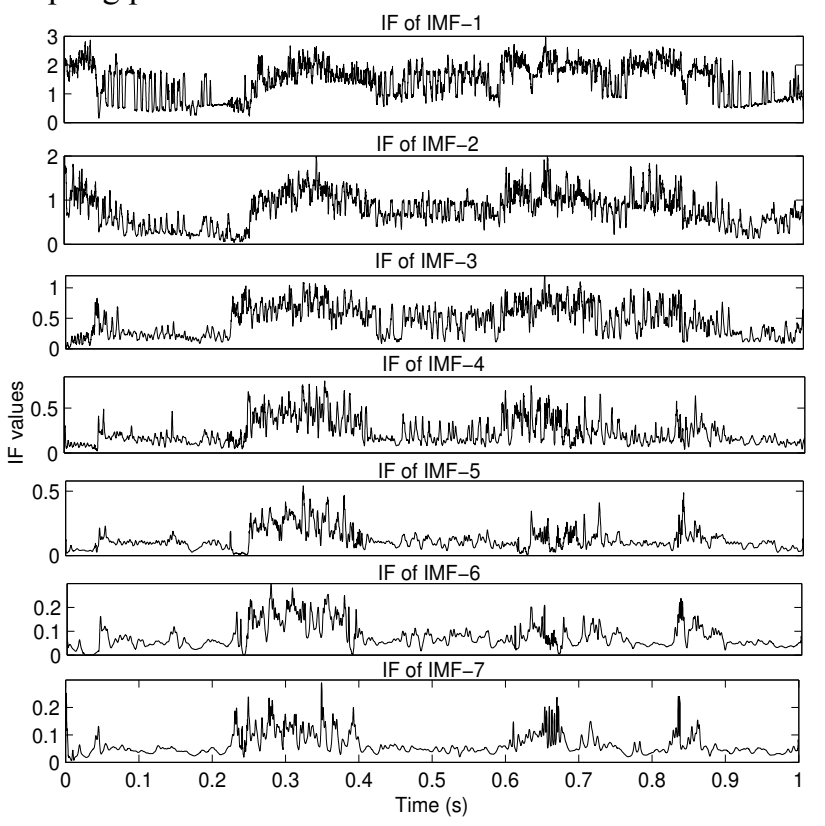

Fig. 2 Instantaneous frequencies of the first 6 IMFs of noisy speech signal shown in Fig. 1

\subsection{Hilbert spectrum}

Hilbert spectrum represents the distribution of the signal energy as a function of time and frequency. It is also designated as Hilbert amplitude spectrum $H(k, t)$ or simply Hilbert spectrum(HS). This process first normalizes the IF values. The number of desired frequency bins is an input parameter to construct HS. The overall HS is expressed as the superposition of the individual IMFs' HSs defined as: $H(k, t)=\sum H^{(b)}(k, t)$ for $b=1,2, \ldots \ldots, B$ [21]. Hence, each element $H(k, t)$ of the overall HS is defined as the weighted sum of the instantaneous amplitudes of all the IMFs at $k^{\text {th }}$ frequency bin.

$$
H(k, t)=\sum_{b=1}^{B} a_{b}(t) w_{b}^{(k)}(t)
$$

where the weight factor $w_{b}^{(k)}(t)$ takes 1 if $a_{b}(t)$ falls within $k$ th band, otherwise it is 0 . After computing the elements over the frequency bins, $\mathrm{H}$ represents the instantaneous signal spectrum in time-frequency space as a $2 \mathrm{D}$ matrix. It is noted that the time resolution of $\mathrm{H}$ is equal to the sampling rate and the frequency resolution can be chosen up to Nyquist limit [26]. Fig. 4 represents the Hilbert spectrum (in $\mathrm{dB}$ ) of the voiced speech signal shown in Fig. 1 using 128 frequency bins. The method of representing the signal in time-frequency domain using Hilbert spectrum obtained by EMD together with Hilbert transform is also called Hilbert-Huang transform (HHT). 


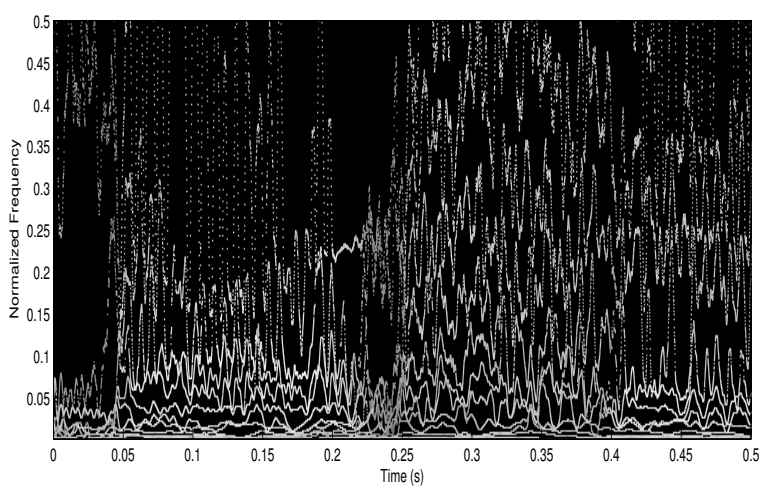

Fig. 3 Hilbert spectrum (HS) of speech signal illustrated in Fig. 1

The marginal spectrum represents the cumulated energy over the entire data span in a probabilistic sense at a frequency index. The marginal power spectra corresponding to the Hilbert spectrum $H(k, t)$ can be defined as:

$$
\psi(k)=\sum_{t=1}^{T} H(k, t)
$$

where $T$ is the total data length. The marginal HS plays a different interpretation rather than Fourier spectra [21]. In the Fourier spectra, the existence of energy at a frequency, $\mathrm{k}$, means a component of a sine or a cosine wave persisted through the time span of the data. The Fourier energy spectrum clearly represents a stack of harmonics. Whereas, the existence of energy in marginal Hilbert spectrum at the frequency, $\mathrm{k}$, means only that, in the whole time span of the data, there is a higher likelihood for such a wave to have appeared locally.

\subsection{Bivariate EMD (bEMD)}

The traditional EMD is only suitable for univariate (real valued) signals. The complex empirical mode decomposition (cEMD) is an extension of the basic EMD suitable for dealing with complex signals [27]. The motivation to extend EMD is that a large number of signal processing applications have complex signals. In addition, this extension is applied on both the real and imaginary parts simultaneously because complex signals have a mutual dependence between the real and imaginary parts. Thus, if the decomposition is done separately, the mutual dependency will be lost.

The bivariate empirical mode decomposition (bEMD) is more generalized extension of EMD to complex signals. The main difference between bEMD and cEMD is that the latter uses the basic EMD to decompose complex signals, whereas bEMD adapts the rationale underlying the EMD to a bivariate framework [28, 29]. In bEMD two variables are decomposed simultaneously based on their rotating properties. The algorithm of bEMD, as proposed in [28], is as follows:

1) For $1<\mathrm{q}<Q$,

a) Project $x(t)$ on direction $\phi_{q}: p_{\phi_{q}}(t)=\operatorname{Re}\left(e^{-j \phi_{q}} x(t)\right)$

b) Extract the maxima of $p_{\phi_{q}}(t):\left(t_{i}^{q}, p_{i}^{q}\right)$ c) Interpolate the set of points $\left(t_{i}^{q}, e^{j \phi_{q}} p_{i}^{q}\right)$ to obtain the partial envelope curve in direction $\phi_{q}$ named $e_{\phi_{q}}(t)$

2) Compute the mean of all tangents:

$$
e(t)=\frac{2}{Q} \sum_{q} e_{\phi_{q}}(t)
$$

3) Subtract the mean to obtain $\hat{\delta}(t)=x(t)-e(t)$

4) Test if $\hat{\delta}(t)$ is an IMF:

- If yes, repeat the procedure from the step 1 on the residual signal.

- If not, replace $x(t)$ with $\hat{\delta}(t)$ and repeat the procedure from step 1.

The bivariate EMD can now be expressed as:

$$
x(t)=\sum_{m} \hat{\delta}_{m}(t)+r_{C}(t)
$$

where $\hat{\delta}_{m}(t)$ denotes the $m^{\text {th }}$ extracted complex empirical mode and $r_{C}(t)$ the residual. bEMD is employed to decompose the speech signals contaminated by additive noise.

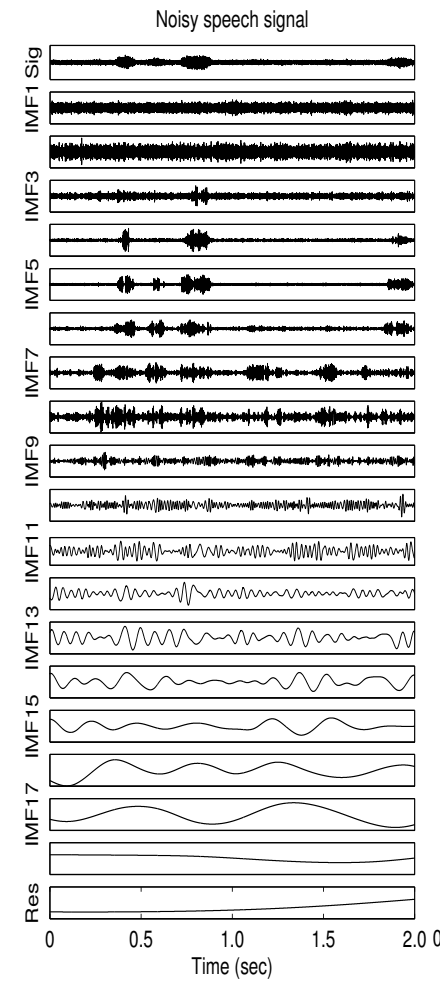

Fractinal Gaussian noise (fGn)

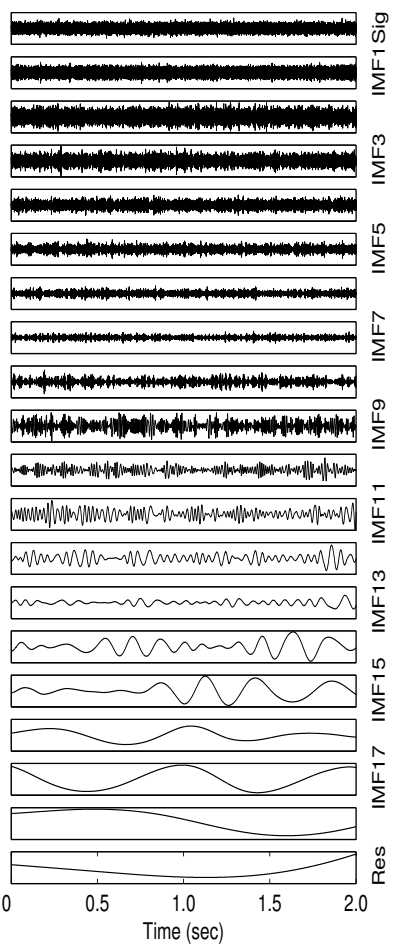

Fig. 4 The illustration of bEMD: A noisy speech signal and fGn are decomposed together using bEMD.

The noisy speech signal and fractional Gaussian noise (fGn) together are decomposed using bEMD as sown in Fig. 4. The normalized fGn as well as the speech signal are considered as real and imaginary parts of the complex signal. It is well known that EMD of fGn acts as dyadic fitrer-bank [23]. With the use of fGn in bEMD, the decomposition of speech signal associated with fGn is considered as dyadic filter-bank. 


\subsection{Multivariate EMD (mEMD)}

The multivariate EMD (mEMD) is more generalized extension of EMD suitable for dealing with direct processing of multivariate data for real world applications [30]. Standard EMD revealed that IMFs tend to mimic a filter bank-like decomposition, similar to wavelet decompositions. Multivariate EMD not only exposes filter bank structure, but also ensures the overlapping of the frequency responses of the filters associated with IMFs of the same index from multiple channels. To extend general idea of multivariate signals for mEMD, input data are straightforwardly processed in $n$-dimensional spaces to generate multiple $n$-dimensional envelopes by taking signal projections along different directions in $n$-dimension spaces. The calculation of the local mean can be considered an approximation of the integral of all the envelopes along multiple directions in an $n$-dimensions space. This step is complex to perform due to the lack of formal definition of maxima and minima in $n$-dimensional domains in general EMD. The sampling based on low discrepancy Hammersley sequence is used to generate projections of input signal in [30]. Once the projections along different directions in multidimensional spaces are obtained, their extrema are interpolated via cubic spline interpolation to obtain multiple signal envelopes. Thus obtained envelopes are then averaged to obtain the local mean of the multivariate signal. The following algorithm proposed in [31] is employed here to decompose signal $s(t)$ into a set of IMF components.

1. Generate the pointset based on the Hammersley sequence for sampling on an (n-1)-sphere [30];

2. Calculate a projection, denoted by $\left.p^{\theta_{k}}(t)\right\}_{t=1}^{T}$, of the input signal $\{s(t)\}_{t=1}^{T}$ along the direction vector $X^{\theta_{k}}$, for all $k$ (the whole set of direction vectors), giving $\left.p^{\theta_{k}}(t)\right\}_{k=1}^{K}$ as the set of projections;

3. Find the time instants $\left.t_{i}^{\theta_{k}}\right\}_{k=1}^{K}$ corresponding to the maxima of the set of projected signals $\left.p^{\theta_{k}}(t)\right\}_{k=1}^{K}$;

4. Interpolate $\left[t_{i}^{\theta_{k}}, s\left(t_{i}^{\theta_{k}}\right)\right]$, for all values of $k$, to obtain multivariate envelope curves $\left.e^{\theta_{k}}(t)\right\}_{k=1}^{K}$;

5. For a set of $K$ direction vectors, calculate the mean $\mu(t)$ of the envelope curves as:

$$
\mu(t)=\frac{1}{k} \sum_{k=1}^{K} e^{\theta_{k}}(t)
$$

6. Extract the "detail" $d(t)$ using $d(t)=X(t)-\mu(t)$. If the "detail" $d(t)$ fulfills the stoppage criterion for a multivariate IMF, apply the above procedure to $X(t)$ $d(t)$, otherwise apply it to $d(t)$.

Consider a sequence of $N$-dimensional vectors $\{s(t)\}_{t=1}^{T}=\left\{s_{I}(t), s_{2}(t), \ldots \ldots \ldots s_{N}(t)\right\}$ representing a multivariate signal with $N$ components, and $X^{\theta_{k}}=\left\{x_{1}^{k}, x_{2}^{k}, \ldots \ldots ., x_{N}^{k}\right\}$ denoting a set of direction vectors along the directions given by angles $\theta^{k}=$ $\left\{\theta_{1}^{k}, \theta_{2}^{k}, \ldots \ldots . ., \theta_{(N-1)}^{k}\right\}$ on an $(n-1)$-sphere.

Once the first IMF is extracted, it is subtracted from the input signal and the same process is applied to the resulting signal yielding the second IMF and so on. In the multivariate case, the residue corresponds to a signal whose projections do not contain enough extrema to form a meaningful multivariate envelope. The stopping criterion for mEMD of IMFs is similar to standard EMD [32], the difference being that the condition for equality of the number of extrema and zero crossings is not imposed, as extrema cannot be properly defined for multivariate signals.

\subsection{Limitations of EMD}

The EMD method has gained some recognition over the past few years. The theoretical base has not been fully established. Up to this time, most of the progress with EMD has been in its applications, while the underlying mathematical problems have been mostly left untreated [33]. All the results have come from case-by-case comparisons conducted empirically. It is producing great results but waiting for mathematical foundation on which to rest its case. We can consider square wave with constant amplitude over time as shown in Fig. 5.

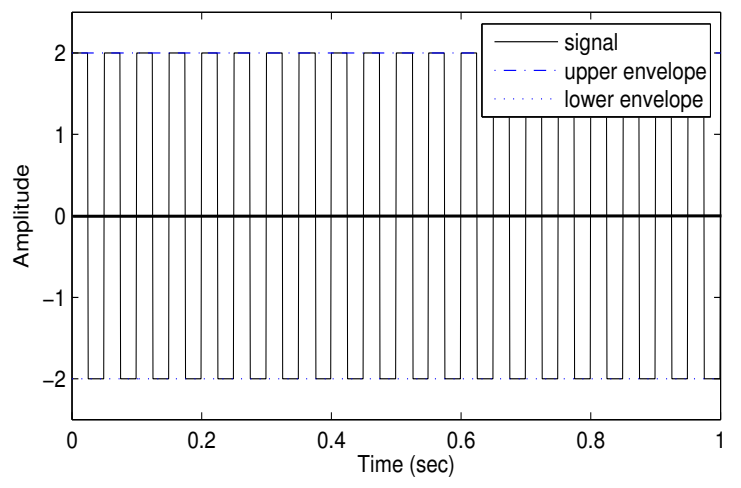

Fig. 5 Square wave with constant amplitude over time

Theoretically, it contains infinite number of harmonics and if it is decomposed using Fourier transform, all the components will be extracted. No decomposition is possible using EMD. The upper and lower envelopes will be the straight lines and their local mean value will zero. The analyzing square wave will be treated as an IMF and hence no more IMF will be produced which does not meet the theoretical hypothesis. Another limitation is that EMD requires high computational power and it is nearly impossible to implement any application with EMD in real time. It is required to understand the underlying decomposition process of EMD before applying it to any data. Any signal can be analyzed by mathematical model based approach i.e. Fourier transformation while it is necessary to investigate the data before applying EMD for multiband decomposition.

\subsection{Comparative study}

Hilbert-Huang Transform (HHT) is obtained by combining EMD and Hilbert spectral analysis. Empirically, all tests indicate that HHT is superior tool for timefrequency analysis of non-linear and non-stationary signals. It is based on the adaptive basis, and the frequency is 
defined through Hilbert transform. There is no need for the spurious harmonics to represent nonlinear waveform deformations as any of the a priori basis methods. There is no uncertainty principle limitation on time or frequency resolution. A comparison of Fourier, wavelet and HHT analysis is summarized in Table 1 .

Table 1 A comparative summary of Fourier, Wavelet and Hilbert-Huang transformations

\begin{tabular}{|l|l|l|l|}
\hline & Fourier & Wavelet & Hilbert \\
\hline Basis & A priori & A priori & Adaptive \\
\hline Frequency & $\begin{array}{l}\text { Global, } \\
\text { uncertainty }\end{array}$ & $\begin{array}{l}\text { Regional, } \\
\text { uncertainty }\end{array}$ & $\begin{array}{l}\text { Local, } \\
\text { certainty }\end{array}$ \\
\hline Presentation & $\begin{array}{l}\text { Energy- } \\
\text { frequency }\end{array}$ & $\begin{array}{l}\text { Energy-time- } \\
\text { frequency }\end{array}$ & $\begin{array}{l}\text { Energy-time- } \\
\text { frequency }\end{array}$ \\
\hline Nonlinear & No & No & Yes \\
\hline Non-stationary & No & Yes & Yes \\
\hline $\begin{array}{l}\text { Theoretical } \\
\text { base }\end{array}$ & $\begin{array}{l}\text { Theory } \\
\text { complete }\end{array}$ & $\begin{array}{l}\text { Theory } \\
\text { complete }\end{array}$ & Empirical \\
\hline
\end{tabular}

\section{Speech Enhancement}

The terms speech enhancement and speech cleaning properly refer respectively to the improvement in the quality or intelligibility of a speech signal and the reversal of degradations that have corrupted it. Although there are numerous methods [22] of speech enhancement, this study focuses on soft-thresholding based approach. The softthresholding is the way to suppress the noise effects from the noisy speech signal by comparing the analyzing signal with a predefined threshold value.

\subsection{Soft-thresholding with DCT (sDCT)}

In the transform domain, speech enhancement methods commonly use amplitude subtraction based soft thresholding defined by $[34,35]$

$$
\hat{X}_{k}=\left\{\begin{array}{cc}
\operatorname{sign}\left(X_{k}\right)\left(\left|X_{k}\right|-\sigma_{v}\right) & \text { if }\left|X_{k}\right|>\sigma_{v} \\
0 & \text { otherwise }
\end{array}\right.
$$

where $\sigma_{v}$ denotes the noise level, $X_{k}$ is the $k$ th coefficient of the noisy signal obtained by the analyzing transformation and $\hat{X}_{k}$ represents the corresponding thresholded coefficient. Since all the coefficients are thresholded by $\sigma_{v}$, the speech components are also degraded during this process. This degradation results in a loss in speech quality. Unlike the conventional constant noise-level subtraction rule in equation Eq. (11), a frame based soft thresholding strategy is proposed in [36]. The strategy depends on segmenting the signal into short time intervals and applying discrete cosine transform (DCT) on each frame. The DCT coefficients of each frame are divided into frequency bins which are categorized as either signal or noise dominant depending on its speech and noise energy distribution. The classification pertains to the average noise power associated with that particular bin. If the $i$ th bin satisfies the following inequality,

$$
\frac{1}{K} \sum_{k=1}^{K}\left|X_{k}^{i}\right|^{2} \geq \sigma_{n}^{2}
$$

where $\sigma_{n}^{2}$ denotes the variance of the noise, $X_{k}^{i}$ is the $k$ th DCT coefficient of the $i$ th frequency bin and $K(=64)$ is the number DCT coefficients of the bin, then the bin is characterized as signal dominant, otherwise as noise dominant. The signal dominant bins are not thresholded, since it is highly possible to degrade the speech signal, especially for high SNRs. In the case of a noise dominant frequency bin, the absolute values of the DCT coefficients are sorted in ascending order and a linear thresholding is applied:

$$
\hat{X}_{k}=\operatorname{sign}\left(X_{k}\right)\left[\max \left\{0,\left(\left|X_{k}\right|-\eta_{j}\right)\right\}\right]
$$

where $\eta_{j}$ is the linear threshold function obtained as,

$$
\eta_{j}=j \frac{\lambda \sigma_{n} K}{\sum_{k=1}^{K} k^{2}}
$$

where $j$ is the index of sorted $\left|X_{k}\right|$. It is evident from Eq. (12) that for the noise-dominant frequency bins, the average noise power added would be less than the average noise power estimated over the entire speech signal. Here, the added average noise power over any of these frequency bins is denoted as $\lambda \sigma_{n}$. The reasonable value of $\lambda(0.35$ to 0.8 [36]) is determined experimentally. Using the categorization in Eq. (12) at each frequency bin, the noise dominants are identified and value of $\lambda$ is calculated by simply dividing the variance of that frequency bin with the overall noise variance.

\subsection{EMD based soft-thresholding (sEMD)}

The data adaptive uEMD method is more suitable to decompose speech, non-stationary signal yielding better performance in soft-thresholding. The modified method is based on applying the soft thresholding algorithm in Eq. (13) to the IMFs of the noisy speech. First, uEMD is applied to the noisy speech. The obtained IMFs are divided into sub-frames (8ms). Similar to the DCT case, these subframes are characterized as either a signal dominant or a noise dominant sub-frame. However, for categorizing the sub-frames, unlike the limit defined in Eq. (12), a different strategy is introduced [37]. This new soft-thresholding strategy provides an effective limit for the sub-frame categorization. Moreover, the noise variance used in thresholding is estimated separately for each IMF. This new strategy is applied to IMFs of the noisy speech signal.

The sub-frames classification into signal and noise dominants is one of the key points of the soft thresholding algorithm. It makes possible to eliminate the noise signals without degrading the original speech components. It is not feasible to use the soft-thresholding directly to IMFs. Each IMF contains different noise and speech energy distribution, which suggests that each IMF will have a different noise and speech variance. Therefore, the noise variance of each IMF should be defined separately and the limit for sub-frame categorization should have a larger value then the limit defined in Eq. (12), in order to guarantee that all the noisy sub-frames are thresholded. A 
novel limit relies on the idea that a sub-frame can be defined as a noise dominant sub-frame, if the noise power is higher than the speech power. Therefore, the limit should be set to the case where the noise and speech variance $\left(\sigma_{s}^{2}\right.$ and $\left.\sigma_{n}^{2}\right)$ are same. The variance $\sigma^{2}$ of the noise contaminated speech sub-frame is represented as,

$$
\sigma^{2}=\sigma_{s}^{2}+\sigma_{n}^{2}+2 \varphi(s, n)
$$

where $\varphi(s, n)$ is the covariance term of signal and noise. If the signal and noise are independent, the covariance function gives zero, thus we have

$$
\sigma^{2}=\sigma_{s}^{2}+\sigma_{n}^{2}
$$

For frame categorization (into signal and noise dominant frames), the threshold is considered with equal noise and speech power, and hence $\sigma^{2}=2 \sigma_{n}^{2}$. Therefore, in case of equal noise and speech power, the variance of the subframe is equal to $2 \sigma_{n}^{2}$. That is why the limit for the categorization of the sub-frame in Eq. (12) should be set to this value. With the proposed strategy, if

$$
\frac{1}{K} \sum_{k=1}^{K}\left|X_{k}^{i}\right|^{2} \geq 2 \sigma_{n}^{2}
$$

where $\sigma_{n}^{2}$ denotes the variance of the noise and $X_{k}^{i}$ is the $k$ th sample of the $i$ th sub-frame. Then sub-frame is categorized as signal dominant, otherwise as noise dominant. Noise dominant sub-frames are thresholded as in Eq. (13) and the noise variance in Eq. (14) is calculated separately for each IMF.
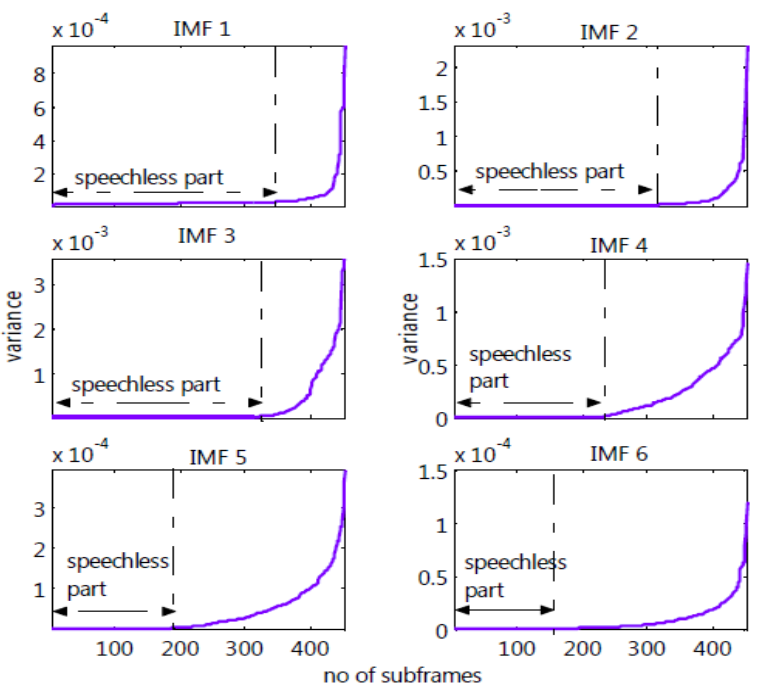

Fig. 6 Sorted noise variance of $8 \mathrm{~ms}$ sub-frames for the first 6 IMFs of a noisy speech signal at $10 \mathrm{~dB}$

The estimation of the variance of each IMF plays an important role in the performance of the EMD soft thresholding algorithm. The calculation is achieved by a data adaptive, efficient algorithm. IMFs are divided into sub-frames (8ms) and the variance of each frame is stored in ascending order in a variance array. Since the speechless parts will mostly have the lowest variance, the noise variance of the sub-frames can be estimated from these speechless parts of the array. Fig. 6 shows a plot of the variance of the sub-frames for the first 6 IMFs of a noisy speech signal at $10 \mathrm{~dB}$. The difference between the noise variance and the length of the speechless parts of IMFs can be observed in Fig. 6. The noise signals are concentrated in the lower order IMFs. The higher order IMFs are mainly contain the speech signals. With this method we have a very good estimation of noise variance of each IMF and the noise components in all the IMFs can effectively be removed.

\subsection{DCT-EMD hybrid soft-thresholding (dctEMD)}

The DCT-EMD hybrid algorithm is based on applying the soft thresholding algorithm in two stages. In the first stage, we use the soft thresholding for DCT enhancement algorithm as a pre-process. As discussed above, this algorithm is effective in removing the noise components for a wide range of SNR values. However, due to the thresholding criteria, the noise signals in the signal dominant sub-frames are not removed. Moreover, a significant amount of the noise signals in the noise dominant sub-frames remains within the signal due to the subtraction rule. Therefore a significant amount of noise still exists in the enhanced signal. This looks like a white noise and results in an irritating sound. It is not an easy task to detect these noise components and to remove them without degrading the speech signal.

It is possible to extract a considerable amount of this residual noise in the second stage from the IMFs of the enhanced speech. Due to the frequency characteristics of uEMD, the noise and speech signals mostly dominate in different IMFs. Mainly, the high frequency noise components centre in the first few ones. Therefore a noticeable amount of high frequency noise components that were in signal dominant bins in the first stage can be identified from the first IMFs of the enhanced speech.
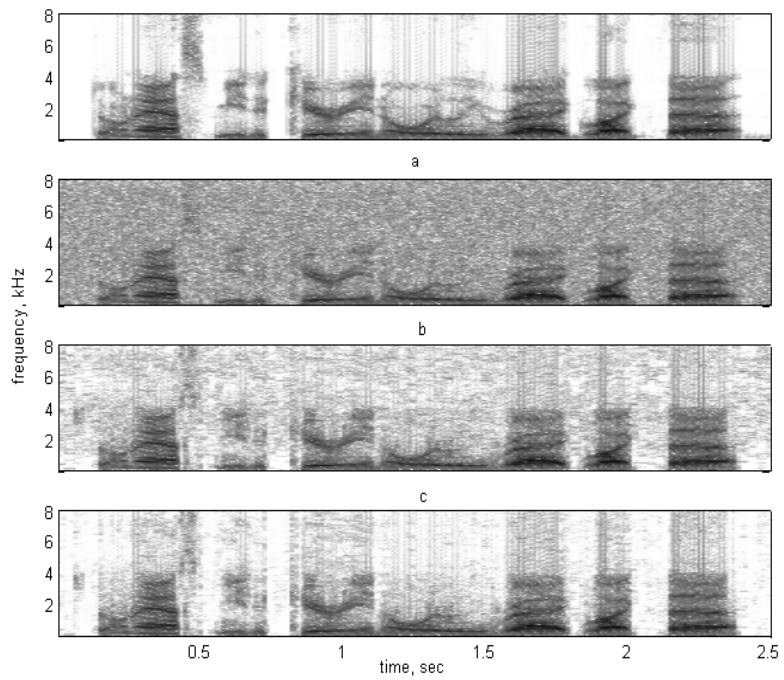

Fig. 7 Spectrograms of (a) Clean speech, (b) Noisy speech corrupted with white noise at $10 \mathrm{~dB}$ SNR, (c) Recovered speech after soft thresholding with sub-band DCT, and (d) Overall recovered speech of the DCT-EMD based hybrid method

Similarly, the lower frequency noise signals can be identified from the later IMFs. The uEMD based softthresholding technique as described in Section 3.2 is applied in the second stage to reduce the noise remaining after the first stage of the hybrid method. The enhancement performance of DCT and DCT-EMD based hybrid 
methods are illustrated in Fig. 7. It is observed that a noticeable amount noise remains after the first stage (DCT soft-thresholding) as shown in Fig. 7(c). In the second stage, EMD based soft-thresholding is applied to suppress the residual noise. The spectrogram of the overall recovered signal in Fig. 7(d) illustrates the effectiveness of our proposed method. We can observe that the spectrogram of the recovered signal is very close to that of the clean speech signal.

\subsection{Adaptive soft-thresholding (ASTh)}

Soft-thresholding strategy proposed by Salahuddin in [36] is a powerful technique of speech enhancement for a wide range of input SNRs. It thresholded only the noise-dominant frames and kept remain the same in case of the signal-dominant frames. The misclassification of frames is a major drawback that causes musical noise [38]. All the frames are processed with a unique noise variance estimated globally from the input speech. Many noise-dominant frames can be identified as signal-dominant due to the fluctuations in the noise variance of the frames when noise energy distribution is not uniform over the speech. The drawbacks of traditional soft-thresholding algorithms are significantly reduced by adaptive thresholding technique. The frame classification criteria described in [36] is modified. The soft thresholding is applied on each IMF. It is known that the thresholding function is dependent on the signal (speech) and noise variances of each IMF. The signal and noise variances are computed for individual IMF. Each IMF is divided into sub-frames and soft thresholding technique is applied on each sub-frame of on the basis of computed variances. The threshold function is computed for individual IMF and hence such thresholding technique is termed as adaptive thresholding. We calculate the noise variance of speech from its silent part of the observed speech signals. The sub-frames are classified as either a speech dominant or a noise dominant based on the noise variance [39]. The adaptive thresholding technique provides an effective boundary for the sub-frame classification. The soft thresholding is carried out on each sub-frame of each IMF adaptively. After properly suppressing noises using soft-thresholding, all IMFs are summed up to obtain the enhanced speech signal.

The value of $\lambda$ is an important factor softthrehsolding and its value is computed adaptively as: $\lambda=\sigma_{n} / \sigma_{f}$, where $\sigma_{n}^{2}$ and $\sigma_{f}^{2}$ represent the variance of globally estimated noise and that of noise added to the sub-frame respectively. In the experiment we use 5 different speech signals (from TIMIT database) of $10 \mathrm{~dB}$ SNR degraded by white noise and the variations of $\lambda$ are illustrated in Fig. 8. It can be observed from Fig. 8 that the value of $\lambda$ varies in between 0.35 to 0.8 for all speech signals. Therefore, the value of $\lambda$ is selected in this range experimentally. The noise variance of individual IMF is determined from the sorted variance array described in Section 3.2) as shown in Fig. 6.

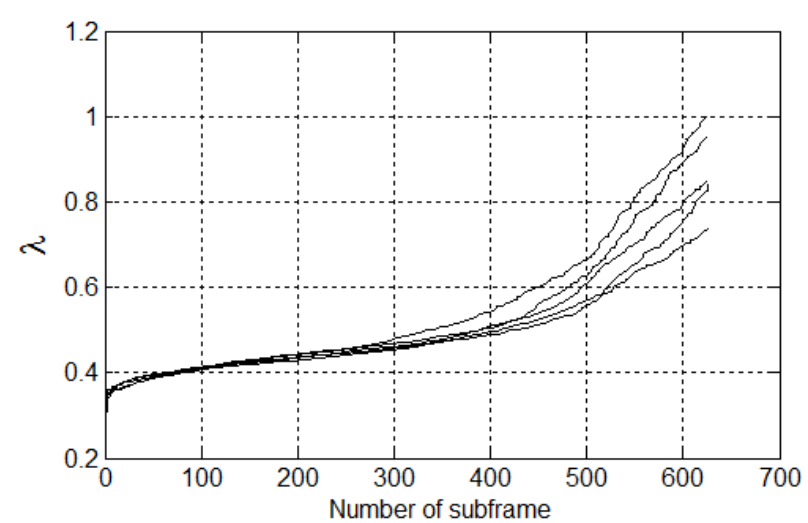

Fig. 8 Estimated values of $\lambda$ in noise dominant subframes

The results of average output SNR using EMD based thresholding algorithm is used to estimate the optimum adaptation factor $\lambda$. In this experiment, the speech signals of English sentences uttered by 7 male and 7 female are randomly selected from TIMIT database. It is observed that the higher value of $\lambda$ is more effective at lower input SNR and lower value for higher input SNR. For this reason, we introduce an expression for the optimum value $\lambda_{\text {opt }}$ of adaptation factor based on the input SNR of the given noisy speech. The derived optimum value of $\lambda$ improves the performance of uEMD based method.

The Input SNR of noisy speech signal is calculated in the similar way of estimating the noise variance of IMFs. The observed speech signal is segmented into frames of length $20 \mathrm{~ms}$ and the variance of each frame is stored in a variance array in ascending order. The noise variance of the noisy speech is estimated from lower (silent) parts of the array. The input SNR can be estimated as:

$$
S N R_{\text {input }}=10 \log _{10}\left(\frac{\sigma_{s+n}^{2}-\sigma_{n}^{2}}{\sigma_{n}^{2}}\right)=10 \log _{10}\left(\frac{\sigma_{s}^{2}}{\sigma_{n}^{2}}\right)
$$

where $\sigma_{s+n}^{2}, \sigma_{s}^{2}$ and $\sigma_{n}^{2}$ are represent the observed, clean and noise variance, respectively. It is found that a specific value of adaptation factor corresponding to an input SNR produces the maximum output SNR. We introduce a formulation to compute the optimum value of $\lambda$ for any given input SNR to achieve maximum speech enhancement. The expression to calculate the optimum adaptation factor $\left(\lambda_{o p t}\right)$ is defined as

$$
\lambda_{\text {opt }}=f(v)=p_{0}+p_{1} v+p_{2} v^{2}+p_{3} v^{3}
$$

to fit the data points $\left(v_{i}, w_{i}\right), i=1,2, \ldots . ., d(=9)$; where $v_{i}$ and $w_{i}$ are the input SNR and optimum value of $\lambda$ (to obtain the maximum output SNR) respectively as listed in Table 2. We experimentally found that there is a nonlinear relation between the input SNR and adaptation factor, for that we choose a third degree polynomial to fit the non-linear data points with minimum stable coefficients [40]. To obtain the coefficients, Eq. (19) can written as $W=V P$ where $W=\left[w_{1}, w_{2}, \ldots, w_{d}\right]^{T}, P=\left[p_{0}, p_{1}\right.$, $\left.p_{2}, p_{3}\right]^{T}$ and $V$ is a matrix with $d$ rows. The $i^{\text {th }}$ row of $V$ can be defined as $V_{i}=\left[\begin{array}{llll}1 & v_{i} & v_{i}^{2} & v_{i}^{3}\end{array}\right]$. The matrix representation $W=V P$ can also be written as $V^{T} W=V^{T} V P$ 
and hence the final expression to find the coefficient vector $P$ is defined as:

$$
P=\left(V^{T} V\right)^{-1} V^{T} W
$$

Equation (20) is solved by using least square method to obtain the values of the coefficients $P=\left[p_{0}, p_{1}, p_{2}, p_{3}\right]^{T}$. Then the value of optimum adaptation factor $\lambda_{\text {opt }}$ can easily be calculated using Eq. (14). It is not necessary to use the only input SNRs listed in Table 2. $\lambda_{\text {opt }}$ can be computed for any given input SNR satisfying the least square fit method.

Table 2 Values of $\lambda$ to obtain maximum output SNR for different input SNRs

\begin{tabular}{|c|c|c|c|c|c|c|c|c|c|}
\hline $\mathrm{SNR}_{\text {input }}$ & -10 & -5 & 0 & 5 & 10 & 15 & 20 & 25 & 30 \\
\hline$\lambda$ & 0.80 & 0.73 & 0.71 & 0.7 & 0.62 & 0.60 & 0.54 & 0.51 & 0.5 \\
\hline
\end{tabular}

\subsection{Noise assisted Soft-thresholding (NaSTh)}

The perfect estimation of noise level is a priori to implement the soft-thresholding based speech enhancement algorithms. In the existing algorithms the noise is usually estimated from the speechless part of the noisy speech signal. Such method degrades the performance of speech enhancement when the speechless is not detected perfectly. Bivariate EMD (bEMD) based approach is implemented for effective noise estimation to derive the parameters required for soft-thresholding [41]. In bEMD, two variables are decomposed simultaneously without losing mutual dependency. The fractional Gaussian noise (fGn) and noisy speech signal are decomposed together using bEMD producing two separate sets of IMFs corresponding to individual signals. It is found that that the lower order (higher frequency) IMFs contributes higher energies in fGn. It is agreed with the assumption that speech signal contains more noise at higher frequency and hence it is justified to use the fGn as the reference signal to determine the noise variance. Moreover, more IMFs are generated when fGn is combined with speech to apply bEMD. The amplitude of fGn is adjusted according to the noise variance of the observed speech signal.

The effective estimation of noise variance of the noisy speech plays a vital role to the performance of the proposed bEMD based speech enhancement algorithm. The speech signal is considered as a smoothly varying signal with additive Gaussian noise of zero mean and both are uncorrelated from each other. It can be transformed through a low order polynomial form as:

$$
f(s)=\sum_{l=0}^{L} a_{l} s^{l}
$$

where $L$ is the degree of polynomial. The purpose of the transformation is to find out the variance of a random variable $y=f(s)$ resulting from the propagation of the noisy speech signal $s$ through the nonlinear polynomial function. The Taylor series expansion is used to obtain exact expression of variance of the random variable distribution [42]. The low order polynomial terms are suppressed using filter based on a finite difference expression. So the initial distribution can be written as: $s=\bar{s}+z$, where $\bar{S}$ is the expectation value of $s$ and $z$ is a zero mean random variable. Then the Taylor expansion for $y$ as

$$
y=f(\bar{s})+\left.\sum_{l=1}^{L} \frac{z^{l}}{l !} \frac{d^{l} f}{d s^{l}}\right|_{s=\bar{s}}
$$

and we can determine the first order moment

$$
\bar{y}=E(y)=f(\bar{s})+\left.\sum_{l=2}^{L} \frac{\vartheta_{l}}{l !} \frac{d^{l} f}{d s^{l}}\right|_{s=\bar{s}}
$$

where $\vartheta_{l}$ denotes the nth order moment of the random variable $z$. The variance of a random variable is its second central moment, the expected value of the squared deviation from the mean [43]. Considering again the Taylor series expansion, the noise variance can be computed as follows

$$
\begin{aligned}
\sigma_{y}{ }^{2} & =E\left[(y-\bar{y})^{2}\right] \\
& =E\left[\left(\left.\sum_{l=1}^{L} \frac{z^{l}}{l !} \frac{d^{l} f}{d s^{l}}\right|_{s=\bar{s}}-\left.\sum_{l=2}^{L} \frac{\vartheta_{l}}{l !} \frac{d^{l} f}{d s^{l}}\right|_{s=\bar{s}}\right)^{2}\right]
\end{aligned}
$$

The overall noise variance thus obtained is used to adjust the energy of fGn prior to the decomposition. Then the derived fGn is combined with the noisy speech to form the complex signal to be decomposed by bEMD.

The observed speech signal $s(t)$ is combined with fGn, $\eta(t)$, yielding the complex signal $x(t)=s(t)+j \eta(t)$. After completion of bEMD, $x(t)$ can be expressed as sum of complex IMFs $\delta(t)$ and final residue $r_{C}(t)$. The real part $a(t)=\operatorname{Re}\langle\hat{\delta}(t)\rangle$ represents IMFs of the speech signal $s(t)$ and the imaginary part $b(t)=\operatorname{Im}\langle\hat{\delta}(t)\rangle$ corresponds to IMFs of $\eta(t)$. Hence, the individual signals can be represented as:

$$
\begin{aligned}
& s(t)=\sum_{m=1}^{M} a_{m}(t)+\operatorname{Re}\left\langle r_{C}(t)\right\rangle \\
& \eta(t)=\sum_{m=1}^{M} b_{m}(t)+\operatorname{Im}\left\langle r_{C}(t)\right\rangle
\end{aligned}
$$

IMFs are divided into frames and then a frame by frame basis the noise variance of $a(t)$ is compared with the variance of $b(t)$ to classify the frames into signal and noise dominants. The variance of $\mathrm{fGn}$ is used here as the reference noise variance. The thresholding is performed only to the noise dominant frames. This process completely overcomes the limitation of computing noise variance from silence part as required in the case of traditional EMD based approach.

The bEMD based speech enhancement algorithm can be summarized as:

i) The overall noise variance is estimated from observed speech signal by Eq. (24) and such variance is used to adjust the amplitude of fGn.

ii) Noisy speech signal and fGn are combined producing complex signal $x(t)$.

iii) bEMD is used to decompose $x(t)$ into complex valued IMFs in which real and imaginary parts correspond IMFs of speech and fGn, respectively.

iv) Each IMF is divided into sub-frames (8ms) to perform very local soft-thresholding. The frame variance of fGn's IMF and energy of the corresponding frame of speech is computed.

v) The frame variance of fGn is used as data adaptive reference energy for binary classification of the 
corresponding speech frame into noise or signal dominant.

vi) Only the noise dominant frames are processed using data adaptive soft-thresholding. The optimum adaptation factor is computed using Eq. (19). The signal dominant frames are left untouched.

vii) All the processed IMFs of speech signals are summed up to obtain the enhanced speech.

Figure 9 shows the spectrogram of the speech cleaned by ASTh and NaSTh as well as the clean and noisy (10dB) speech signals. It is observed that some speech degradation is occurred with ASTh while NaSTh does not make such loss of speech information.

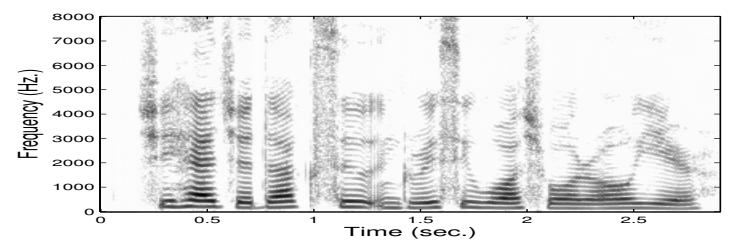

(a)

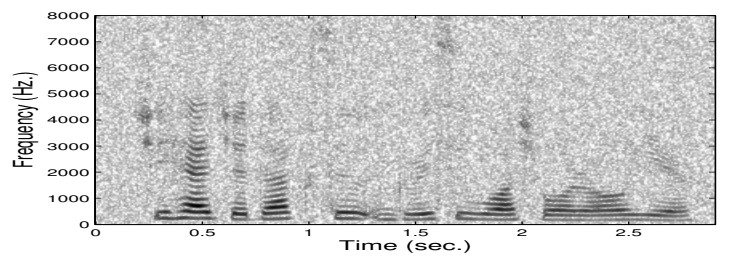

(b)

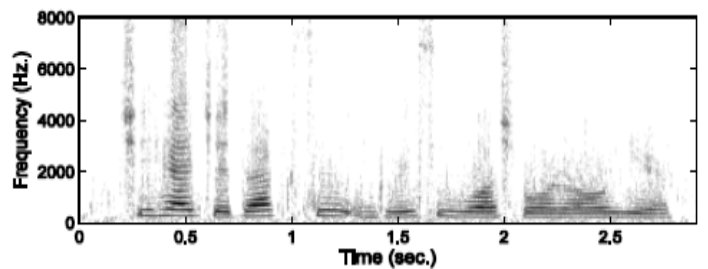

(c)

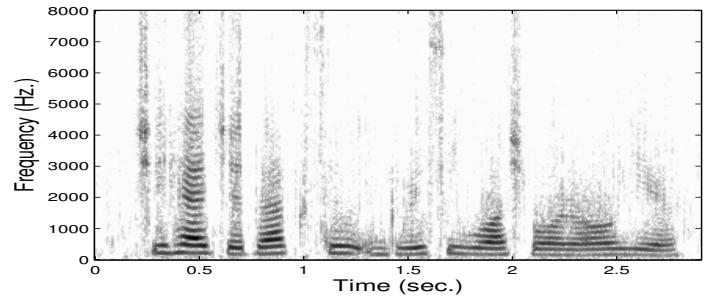

(d)

Fig. 9 Spectrogram of (a) Clean speech, (b) Noisy speech corrupted with white noise at $10 \mathrm{~dB}$ SNR, (c) Recovered speech after adaptive soft-thresholding with ASTh, (d) Enhanced speech using bEMD based NaSTh

\section{Speech Analysis}

The speech signal processing with EMD is recently taken into attention due to its data adaptive nature. The speech signal is being non-stationary, there are limited applications of traditional priori basis based analysis methods i.e. Fourier and wavelet transformation. The voiced/unvoiced speech classification and fundamental frequency estimation are two important issues. These issues implemented using EMD are discussed here.

\subsection{Voiced/unvoiced speech classification}

The efficient classification of short time speech signal into voiced and unvoiced is a crucial preprocessing step in many speech processing applications and is essential in most analysis and synthesis system. The essence of classification is to determine whether the speech production system involves the vibration of the vocal cords. The speech signal originated from the speaker's vocal cords contains a sequence of periodic correlation. Such signal is also called voiced speech signal and unvoiced with absence of periodically correlated sequences. The voiced-unvoiced (V/Uv) discrimination problem is an important one and has been worked on extensively during the last three decades [44].

The HHT based voiced/unvoiced speech classification is performed in [20]. EMD is used to filter the additive noise with the speech signal. The normalized autocorrelation of the filtered speech signal is computed to enhance the periodicity if any. Two features: the energy ratio of the higher frequency band to that of the lower bands and the zero-crossing rate (ZCR) computed in autocorrelation domain are used in classification. The mentioned energy ratio and the ZCR both are lower for the voiced speech signal and that is relatively higher for the unvoiced speech signals. Fig. 10 shows the voiced and unvoiced speech signals and its two (energy based and ZCR) features for each segment. There is a clear correlation between the behaviors of the two features, providing strong basis for marking voiced and unvoiced regions within the signal.
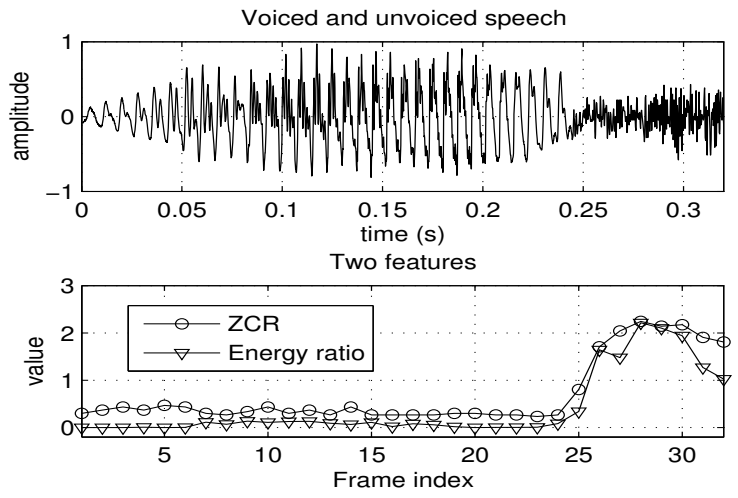

Fig. 10 Voiced-unvoiced speech signals and its corresponding features pair

The marginal Hilbert spectrum (MHS) derived from Hilbert-Huang transformation (HHT) representing precise spectra of the signal is employed to derive the energy based feature. It performs better than that of the Fourier based spectrum. Although this method produces noticeable enhancement of $\mathrm{V} / \mathrm{Uv}$ discrimination performance, it requires training to determine the threshold classification.

A robust voiced/unvoiced classification method by using linear model of empirical mode decomposition (EMD) controlled by Hurst exponent is implemented in [45]. uEMD decomposes any signals into IMFs. It is assumed that voiced speech signal is composed of trend due to vocal cord vibration and some noises. No trend is present in unvoiced speech signal but in voiced one. It is 
considered that the first IMF contains only the noise. A linear model is developed that IMF. Then a specified confidence interval of the linear model is set as the data adaptive energy threshold [46]. There exists higher energy trend in the voiced speech signal and no trend is detected in unvoiced speech. If there exists at least one IMF exceeding the threshold and its fundamental period is within the pitch range, the speech is classified as voiced and unvoiced otherwise. The EMD based algorithm is summarized here:

a. The noisy speech signal is decomposed using uEMD. The first IMF is considered as noise signal

b. Linear noise model [46] is derived on the basis of energy of the first IMF. Its upper limit of $99 \%$ confidence interval is defined as threshold.

c. Construct a set $\Gamma$ of IMFs whose energies exceed the threshold. Determine the fundamental periods of those IMFs.

d. If the fundamental period of least one IMF in $\Gamma$ is within the pitch range, the speech segment is classified as voiced and unvoiced otherwise [45].

The energy distribution of voiced and unvoiced speech signals along with the corresponding noise models are illustrated in Fig. 11 and Fig. 12, respectively. The superiority of the algorithm is that it does not require any training data. The threshold is determined in an adaptive way on the basis of the current speech frame.

(a)
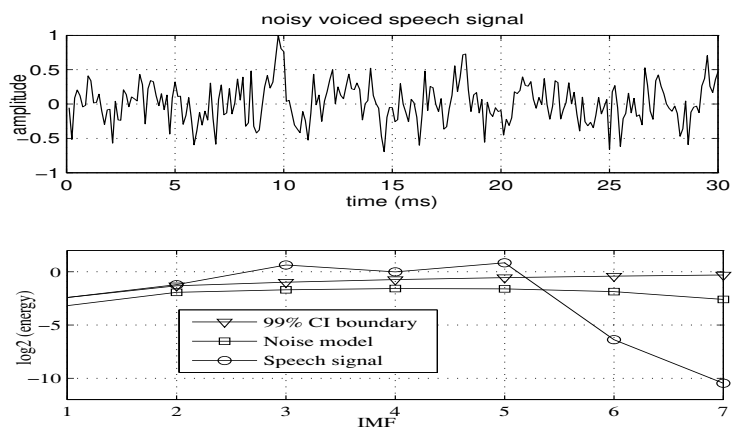

(b)

Fig. 11 (a) Noisy speech signal (0dB SNR), (b) Energies of IMFs 3-5 exceed the threshold of $99 \%$ confidence interval and hence they collectively represent the trend of the signal

(a)

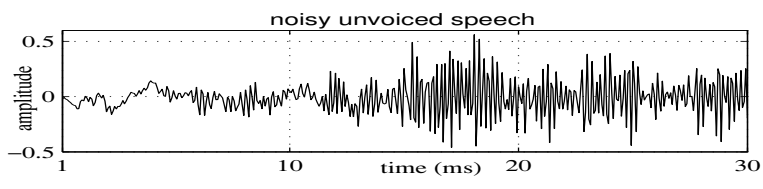

(b)

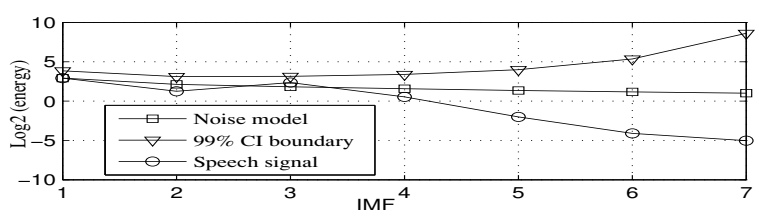

Fig. 12 (a) Unvoiced speech signal of 0dB SNR, (b) Trend detection scenario with unvoiced signal: There is no IMF whose energy exceeds the upper limit of $99 \%$ confidence interval and hence the speech is unvoiced.

\subsection{Pitch estimation}

Pitch, the auditory attribute of speech signal, is an important parameter that is often determined to measure the superiority of performance of many speech processing algorithms [47]. The estimation of pitch period plays an important role in different speech processing applications including speech enhancement using harmonic model, automatic speech recognition and understanding, analysis and modeling of speech prosody, low-bit-rate speech coding etc. There are many pitch estimation algorithms available now-a-days. Different algorithms have been implemented in the time domain $[48,49]$ but none of them meets the desired performance of pitch estimation. The pitch estimation is also performed in the transformed domain. The transformation is usually achieved by traditional Fourier [50] and wavelet transforms [51]. The speech signal is being non-stationary these transformations are not suitable enough. As a data adaptive signal analysis tool EMD is recently used for pitch estimation [19, 52, 53].

An efficient algorithm for pitch determination is implemented in [54] using combination of dominant harmonic modification and EMD. Its basic idea is to reshape the speech signal using a combination of the dominant harmonic modification (DHM) and data adaptive time domain filtering techniques. The noisy speech signal is filtered within the ranges of fundamental frequencies $(50-500 \mathrm{~Hz})$ to obtain the pre-filtered signal (PFS). The dominant harmonic (DH) of the PFS is determined and enhanced its amplitude. The dominant harmonic is the frequency with maximum energy in Fourier domain. Normalized autocorrelation function (NACF) is applied to that modified signal. Then empirical mode decomposition (EMD) based data adaptive time domain filtering is applied to the NACF signal. Partial reconstruction is performed in EMD domain. The pitch period is determined from the partially reconstructed signal. The performance of the EMD based algorithm is better than the other recently developed methods for noisy and clean speech signals. The proposed algorithm for pitch estimation is as follows:
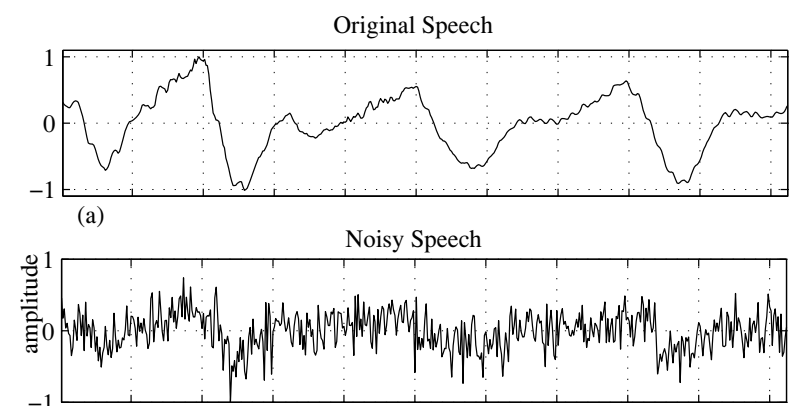

(b) Pre-Filtered Speech

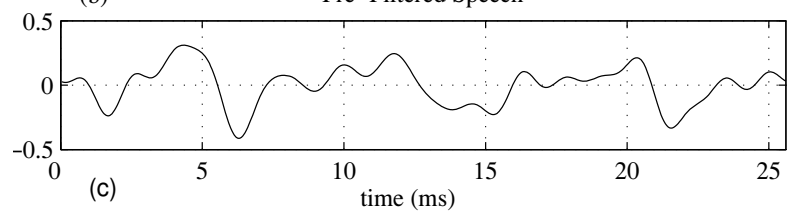

Fig. 13 (a) Clean speech, (b) Noisy speech (0dB SNR) and (c) Pre-filtered speech (PFS)

- Apply pre-filtering to the noisy speech signal to remove a significant portion beyond the pitch range 
$50-500 \mathrm{~Hz}$. It is termed as pre-filtered speech (PFS) as shown in Fig. 13.

- Perform signal modification using dominant harmonic enhancement [55] to the PFS signal.

- Compute NACF of the modified signal

- Apply EMD to NACF of the modified signal shown in Fig. 14.
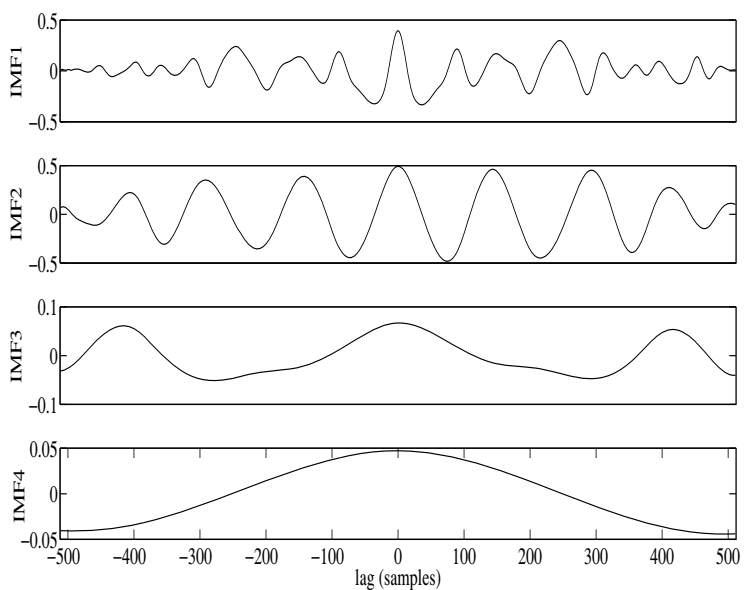

Fig. 14 EMD of NACF with DHM of signal shown in Fig. 13

- Sum up IMFs whose fundamental period lies within the specified pitch range for partial reconstruction of NACF of the modified PFS. The original and partially reconstructed signals are shown in Fig. 15.
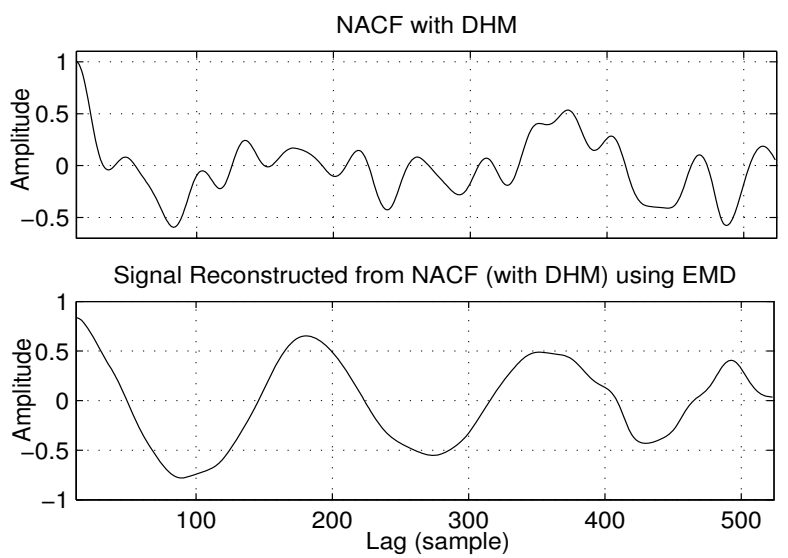

Fig. 15 NACF of the signal after performing the DH enhancement (top), the partially reconstructed signal from NACF with DHM using EMD (bottom): The $2^{\text {nd }}$ and $3^{\text {rd }}$ IMFs are added for partial reconstruction.

- Take the right half of the reconstructed signal.

- The amplitude at zero-lag is selected as the starting index of the pitch period.

- Find the next highest peak from the right half of the reconstructed signal.

- Calculate the pitch period from the difference between the starting index and the next highest peak index. The performances of different pitch estimation algorithms are illustrated in Fig. 16.

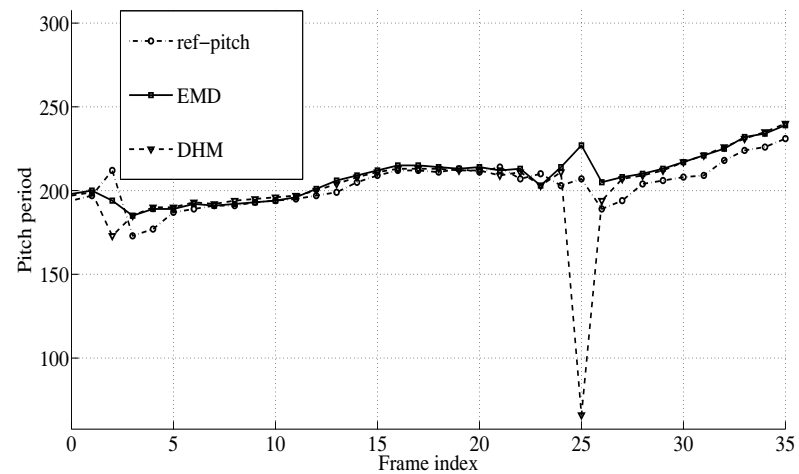

Fig. 16 Comparison of preliminary pitch period estimated by EMD (proposed) and DMH [55] methods with the reference pitch for male speech: The proposed EMD based method performs better than DHM.

\section{Conclusions}

A detail study on advanced speech signal processing using EMD is presented here. It consists of EMD based soft-thresholding, voiced/unvoiced speech discrimination and effective pitch estimation of noisy speech signals. The soft-thresholding based speech enhancement is a potential approach to speech enhancement which is usually performed in the transformed domain rather than the original time domain of the noisy speech signals. The discrete cosine transform and wavelet transform are widely used in soft-thresholding. These transformations use priori basis functions which are not suitable for non-stationary speech signal. EMD is suitable to decompose any nonstationary and non-linear signal into a finite set of subband signals. It is fully data adaptive and does not require any priori basis function. Different soft-thresholding algorithms based on EMD are developed recently with higher performance in speech enhancement. The perfect detection of threshold value is a crucial factor to obtain better performance in soft-thresholding based algorithm. The adaptation factor $\lambda$ is an important term which is used to weight the threshold. Several algorithms (sEMD, dctEMD) use constant value of $\lambda$ and some algorithms (ASTh, $\mathrm{NaSTh}$ ) employ the value of $\lambda$ adapted with input SNR. The latter category performs better.

EMD is recently used in advanced speech signal analysis. The voiced/unvoiced speech discrimination is an important issue in speech analysis. It is usually performed using different acoustic features. An extensive training is required to determine the threshold for classification. The data adaptive EMD based approach is introduced for $\mathrm{V} / \mathrm{Uv}$ classification without any training. The threshold value is adaptively determined in each speech segment. It is more effective in real world application. Another important speech analysis technique is the perfect estimation of fundamental frequency (pitch). The error in pitch estimation is usually occurred due to the half-pitch and double-pitch errors. An EMD based pre-filtering approach is recently introduced [55] to reduce such errors and hence better performance is achieved.

EMD requires very high computational power which does not support to implement online processing system. It is suitable when only for the case of offline processing. It is fully reversible method. When the system requires the reconstruction of decomposed signal, EMD performs better. 
It has very poor capability of frequency domain representation of the analyzing signal. One should think about the mentioned important characteristics of EMD before considering it for any application. The research community of single channel speech enhancement will be greatly benefited from this study.

\section{References}

[1] P. Flandrin: Time-Frequency/Time-Scalfe Analysis, Academic Press, 1999.

[2] K. Grochenig: Foundation of Time-Frequency Analysis, Birkhauser, 2001.

[3] H. Tong: Nonlinear Time Series Analysis, Oxford University Press, 1990.

[4] H. Kantz and T. Schreiber: Nonlinear Time Series Analysis, Cambridge University Press, 1999.

[5] C. Diks: Nonlinear Time Series Analysis: Methods and Applications, World Scientific Press, 1999.

[6] B. Widrow and S. D. Stearns: Adaptive Signal Processing, Prentice Hall, 1985.

[7] N. E. Huang, S. R. Long and Z. Shen: The mechanism fir frequency down-shift in nonlinear wave evolution, Adv. Appl. Mech., Vol. 32, pp. 59-111, 1996.

[8] N. E. Huang et al.: The empirical mode decomposition and Hilbert spectrum for nonlinear and non-stationary time series analysis, Proc. R. Soc. London, Ser. A, Vol. 454, pp. 903-995, 1998.

[9] N. E. Huang, Z. Shen and S. R. Long: A new view of water waves - The Hilbert spectrum, Annu. Rev. Fluid Mech., Vol. 31, pp. 417-457, 1999.

[10] M. Datig and T. Schlurmann: Performance and limitations of the Hilbert-Huang transformation (HHT) with an application to irregular water waves, Ocean Eng., Vol. 31, No. 14, pp. 1783-1834, 2004.

[11] Y. Chen and M. Q. Feng: A technique to improve the empirical mode decomposition in the Hilbert-Huang transform, Earthquake Engineering and Engineering Vibration, Vol. 2, No. 1, pp. 75-85, 2003.

[12] M. K. I. Molla and K. Hirose: Multiband linear prediction of speech signals with adaptive order using empirical mode decomposition, Journal of Signal Processing, Vol. 11, No. 6, pp. 471-479, 2007.

[13] K.-H. Wu, C.-P. Chen and B.-F. Yeh: Noise-robust speech feature processing with empirical mode decomposition, EURASIP Journal on Audio, Speech, and Music Processing, Vol. 2011, pp. 1-9, 2011.

[14] A. Pigorini et. al.: Time-frequency spectral analysis of TMS-evoked EEG oscillations by means of Hilbert-Huang transform, J Neurosci Methods, Vol. 198, No. 2, pp. 236$245,2011$.

[15] J. I. Salisbury1 and M.Wimbush: Using modern time series analysis techniques to predict ENSO events from the SOI time series, Nonlinear Processes in Geophysics, Vol. 9, pp. 341-345, 2002.

[16] J. C. Chang, M. Y. Huang, J. C. Lee, C. P. Chang and T. M. Tu: Iris recognition with an improved empirical mode decomposition method, Optical Engineering Vol. 48, No. 4, pp. 047007-15, 2009.

[17] J. Han and M. van der Baan: Empirical mode decomposition for seismic time-frequency analysis, Geophysics, Vol. 78, No. 2, pp. 9-19, 2013.
[18] A. Karagiannis and P. Constantinou: Noise components identification in biomedical signals based on empirical mode decomposition, Int. Conf. on Information Technology and Applications in Biomedicine, pp. 1-4, 2009.

[19] H. Huang and J. Pan: Speech pitch determination based on Hilbert-Huang transform, Signal Processing, Vol. 86, No. 4, pp. 792-803, 2006.

[20] M. K. I. Molla and K. Hirose: Robust voiced/unvoiced classification of speech signals using Hilbert-Huang transformation, Journal of Signal Processing, Vol. 12, No. 6, pp. 473-482, 2008.

[21] M. K I Molla, K. Hirose and N. Minematsu: Separation of mixed audio signals by decomposing Hilbert spectrum with modified EMD, IEICE Trans. Fundamentals of Electronics, Comm. and Computer Science, Vol. E89-A, No. 3, pp. 227-234, 2006.

[22] M. K. I. Molla and K. Hirose: Single mixture audio source separation by subspace decomposition of Hilbert spectrum, IEEE Trans. Audio, Speech, and Language Processing, Vol. 15, No. 3, pp: 893-900, 2007.

[23] P. Flandrin, G. Rilling and P. Gonqalves: Empirical mode decomposition as a filter bank, IEEE Signal Processing Letters, Vol. 11, No. 2, pp. 112-114, 2004.

[24] B. Z. Wu and N. E. Huang: A study of the characteristics of white noise using the empirical mode decomposition method, Proc. Roy. Soc. London A, Vol. 460, pp. 15971611, 2004.

[25] D. G. Long: Comments on Hilbert transform based signal analysis, MERS Technical Report, Id: MERS 04-001, Brigham Young University, USA, 2004.

[26] N. E. Huang, et al.: Application of Hilbert-Huang transform to non-stationary financial time series analysis, Applied Stochastic Model in Business and Industry, Vol. 19, pp. 245-268, 2003.

[27] T. Tanaka and D. P. Mandic: Complex empirical mode decomposition, IEEE Signal Processing Letters, Vol. 14, No. 2, pp. 101-104, 2007.

[28] G. Rilling, P. Flandrin, P. Gonc, alves and J. Lilly: Bivariate empirical mode decomposition, IEEE Sig. Proc. Lett., Vol. 14, No. 12, pp. 936-939, 2007.

[29] M. U. Altaf, T. Gautama, T. Tanaka and D. P. Mandic: Rotation invariant complex empirical mode decomposition, Proc. of IEEE Int. Conf on Acoust. Speech and Signal Proc. (ICASSP), Vol. III, pp. 1009-1012, 2007.

[30] N. Rehman and D. P. Mandic: Multivariate empirical mode decomposition, Proc. of the Royal Society A, Vol. 466, pp. 1291-1302, 2010.

[31] N. Rehman and D. P. Mandic: Filter bank property of multivariate empirical mode decomposition, IEEE Trans. Signal Processing, Vol. 59, pp. 2421-2426, 2011.

[32] N. E. Huang et. at.: A confidence limit for the empirical mode decomposition and Hilbert spectral analysis, Proc. R. Soc. Lond. A, Vol. 459, pp. 2317-2345, 2003.

[33] N. E. Huang and S. R. Long: Normalized Hilbert transform and instantaneous frequency, NASA Patent Pending, GSC 14, 673-1, 2003.

[34] D. L. Donoho: De-noising by soft thresholding, IEEE Trans. Inf. Theory, Vol. 41, pp. 613-627, 1995.

[35] M. Bahoura, and J. Rouat: Wavelet speech enhancement based on the teager energy operator, IEEE Signal Proc. Lett., Vol. 8, pp. 10-12, 2001.

[36] S. Salahuddin, S. Z. Al Islam, M. K. Hasan and M. R. Khan: Soft thresholding for DCT speech enhancement, Electronics Letters, Vol. 38, pp. 1605-1607, 2002.

[37] E. Deger, M. K. I. Molla, K. Hirose, N. Minematsu and M. K. Hasan: EMD-based soft-thresholding for speech enhancement, Proc. of INTERSPEECH, pp.810-813, 2007. 
[38] E. Deger, M. K. I. Molla, K. Hirose, N. Minematsu and M. K. Hasan: Speech enhancement using soft thresholding with DCT-EMD based hybrid algorithm, Proc. of EUSIPCO, pp. 75-79, 2007.

[39] S. Das, M. E. Hamid, K. Hirose and M. K. I. Molla: Singlechannel speech enhancement by NWNS and EMD, Signal Processing: An International Journal (SPIJ), Vol. 3, No. 5, pp. 279-291, 2010.

[40] M. E. Hamid, S. Das, K. Hirose and M. K. I. Molla: Speech enhancement using EMD based adaptive soft-thresholding (EMD-ADT), Int. Journal of Signal Processing, Image Processing and Pattern Recognition Vol. 5, No. 2, pp. 1-16, 2012.

[41] M. E. Hamid, M. K. I. Molla, X. Dang and T. Nakai: Single channel speech enhancement using adaptive softthresholding with bivariate EMD, ISRN Signal Processing, Vol. 2013, pp. 1-9, 2013.

[42] M. B. Luca, S. Azou, G. Burel and A. Serbanescu: On exact Kalman filtering of polynomial systems, IEEE Trans. Circuits and Systems I, Vol. 53, No. 6, pp. 1329-1340, 2006.

[43] A. Kagan and L. A. Shepp: Why the variance?, Statistics and Probability Letters, Vol. 38, No. 4, pp. 329-333, 1998.

[44] S. Ahmadi and A. S. Spanias: Cepstrum-based pitch detection using a new statistical V/UV classification algorithm, IEEE Trans. Speech Audio Pro., Vol. 7 No. 3, pp. 333-338, 1999.

[45] M. K. I. Molla, K. Hirose, S. K. Roy and S. Ahmad: Adaptive thresholding approach for robust voiced/unvoiced classification, Proc. of IEEE Int. Sympo. on Circuits and Systems (ISCAS), pp. 2409-2412, 2011.

[46] G. Rilling, P. Flandrin and P. Goncalves: Detrending and denoising with empirical mode decomposition, Proc. of EUSIPCO, pp. 1581-1584, 2004.

[47] W. Hess: Pitch Determination of Speech Signals: Algorithms and Devices, Springer, Berlin, 1983.

[48] K. Kasi and S. A. Zahorian: Yet another algorithm for pitch tracking, Proc. of IEEE Int. Conf on Acoust. Speech and Signal Proc. (ICASSP), pp.361-364, 2002.

[49] T. Shimamura and H. Kobayashi: Weighted autocorrelation for pitch extraction of noisy speech, IEEE Trans. Speech and Audio Proc., Vol. 9, No. 7, pp. 727-730, 2001.

[50] J. C. Brown and M. S. Puckette: A high resolution fundamental frequency determination based on phase changes of the Fourier transform, J. Acoust. Soc. Am. Vol. 94, No. 2, pp. 662-667, 1993.

[51] N. A. Kader: Pitch detection algorithm using a wavelet correlation model, Proc. of Radio Science Conference, pp. C33/1 - C33/8, 2000.

[52] M. K. I. Molla, K. Hirose, N. Minematsu and M. K. Hasan: Pitch estimation of noisy speech signals using empirical mode decomposition, Proc. of EUROSPEECH, pp. 16451648, 2007.

[53] Z. Yang, D. Huang and L. Yang: A novel pitch period detection algorithm based on Hilbert-Huang transform, LNCS 3338, Sinobiometrics, pp. 586-593, 2004.

[54] S. K. Roy, M. K. I. Molla, K. Hirose and M. K. Hasan: Harmonic modification and data adaptive filtering based approach to robust pitch estimation, International Journal of Speech Technology (Springer), Vol. 14, pp. 339-349, 2011.

[55] M. K. Hasan et. al.: Signal reshaping using dominant harmonic for pitch estimation of noisy speech, Signal Processing, Vol. 86, No. 5, pp. 1010-1018, 2005.

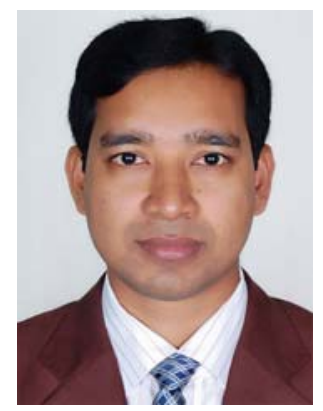

Md. Khademul Islam Molla received his B.Sc. and M.Sc. degrees in electronics and computer science from Shahjalal University of Science and Technology, Bangladesh, in 1995 and 1997, respectively. He obtained his Ph.D. degree from the Department of Frontier Informatics under the Graduate School of Frontier Sciences, The University of Tokyo, Tokyo, Japan in 2006. He joined as an Associate Professor in the Department of Computer Science and Engineering of the University of Rajshahi, Bangladesh, in August 2006, and he has been a Professor since May 2012. From September 2006 to September 2008, he was working as JSPS Postdoctoral Research Fellow in the Department of Information and Communication Engineering, The University of Tokyo, Tokyo, Japan. He was a research fellow at the University of Alberta, Canada, from Nov. 2010 to Oct. 2011. He visited several universities in Japan as guest researcher. His research interests include audio signal processing, blind source separation, braincomputer interface (BCI), biomedical signal and image processing. He is a member of the Institute of Electrical and Electronics Engineers (IEEE). In 2007, he received the Best Paper Award from the Research Institute of Signal Processing, Japan (RISP).

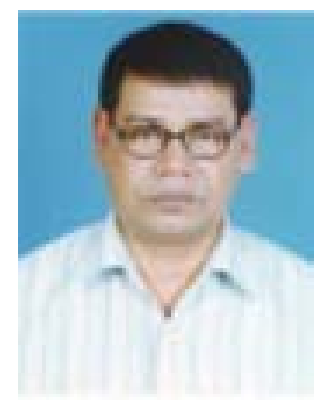

Somlal Das received his B.Sc. and M.Sc. degrees from the Department of Applied Physics and Electronics, Rajshahi University, Bangladesh in 1993 and 1995, respectively. During 1998-2001, he was a Lecturer in the Department of Computer Science and Engineering, Rajshahi University. He obtained his Ph.D. degree from the Department of Computer Science and Engineering, Rajshahi University, Rajshahi, Bangladesh in 2011. He is working as an Associate Professor in the same Department from 2010. His research interests include digital signal processing and speech enhancement.

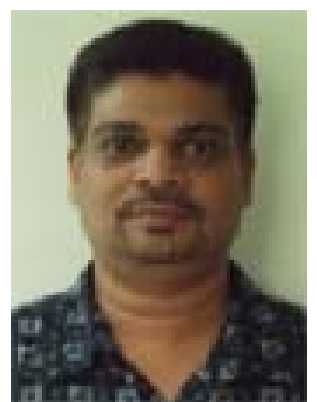

Md. Ekramul Hamid received his B.Sc. and M.Sc. degrees from the Department of Applied Physics and Electronics, Rajshahi University, Bangladesh in 1992 and 1994, respectively. After that he obtained his M.Sc. degree in computer science from Pune University, India in 2002. $\mathrm{He}$ received his Ph.D. degree from Shizuoka University, Japan in 2007. During 1997-2000, he was a Lecturer in the Department of Computer Science and Engineering, Rajshahi University. Dr. Hamid worked as an Assistant Professor at the King Khalid University, Abha, KSA from 2009 to 2011. He is working as an Associate Professor in the Department of Computer Science and Engineering, Rajshahi University from June, 2007. His research interests include digital signal processing, analysis and synthesis of speech signal, speech enhancement and image processing. 


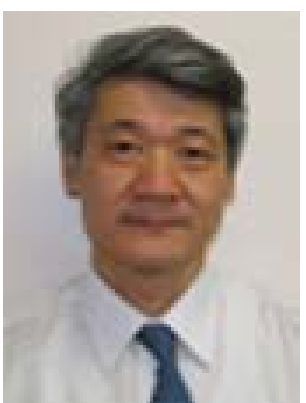

Keikichi Hirose received his B.E. degree in electrical engineering in 1972, and his Ph.D. degree in electronic engineering in 1977, respectively, from the University of Tokyo, Tokyo, Japan. In 1977, he joined the University of Tokyo as a Lecturer in the Department of Electrical Engineering, and, in 1994, became a Professor in the Dept. of Electronic Engineering. From 1996, he was a Professor at the Graduate School of Engineering, Department of Information and Communication Engineering, the University of Tokyo. On April 1, 1999, he moved to the University's Graduate School of Frontier Sciences (Department of Frontier Informatics), and again moved to Graduate School of Information Science and Technology (Department of Information and Communication Engineering) on October 1, 2004. From March 1987 to January 1988, he was a Visiting Scientist of the Research Laboratory of Electronics, Massachusetts Institute of Technology, Cambridge, U.S.A. His research interests cover widely spoken language information processing. He led a project "Realization of advanced spoken language information processing from prosodic features," Scientific Research on Priority Areas, Grant in Aid on Scientific Research, Ministry of Education, Culture, Sports, Science and Technology, Japanese Government. $\mathrm{He}$ is a member of the Institute of Electrical and Electronics Engineers, the Acoustical Society of America, the International Speech Communication Association, the Institute of Electronics, Information and Communication Engineers (Fellow), the Acoustical Society of Japan, and other professional organizations. In 2007, 2012, and 2013, he received the Best Paper Awards from the Research Institute of Signal Processing, Japan (RISP).

(Received October 10, 2013; revised October 23, 2013) 